\title{
Memoria cultural etnobotánica en la vereda El Hatillo, cabecera municipal El Paso, Departamento del Cesar-Colombia
}

\author{
Ethnobotany cultural memory in the EI Hatillo, EI Paso county seat, Cesar's \\ Department-Colombia
}

\section{Memória cultural etnobotânica no município "El Paso" na comunidade rural "El Hatillo", Estado do Cesar-Colômbia}

\author{
Dino Carmelo Manco-Jaraba ${ }^{1}$, Juan Martínez-Martínez², Anderson Duarte-Blanco ${ }^{3}$
}

Forma de citar: D. C. Manco-Jaraba, J. Martínez-Martínez, A. Duarte-Blanco, "Memoria cultural etnobotánica en la vereda El Hatillo, cabecera municipal El Paso, Departamento del Cesar-Colombia”, Respuestas, vol. 20, no. 2, pp. 73-81, 2015.

Recibido:

Diciembre 10 de 2014

Aceptado:

Abril 28 de 2015
${ }^{1}$ Especialización en Ingeniería Ambiental dinomancojaraba@gmail.com ORCID: 0000-0001-8506-094X Fundación Universitaria del

Área Andina

Valledupar-Colombia

${ }^{2}$ Ingeniería de Minas juanmtz@hotmail.com ORCID: 0000-0003-0369-1460

Fundación Universitaria del

Área Andina

Valledupar-Colombia

${ }^{3}$ Ingeniería de Minas anduar22@hotmail.com ORCID: 0000-0002-7798-2079

Fundación Universitaria del

Área Andina

Valledupar-Colombia

\section{Resumen}

Antecedentes: La etnobotánica estudia los saberes producidos por la interacción entre la sociedad y el uso de las plantas que han pasado en generaciones y que aún se conservan en los diferentes sectores de la comunidad rural de la vereda El Hatillo, Jurisdicción La Loma de Calentura, Municipio de El Paso, Cesar. En la actualidad los estudios etnobotánicos realizados en Colombia son muy pocos tales como: Estudios etnobotánicos en los Andes centrales: Distribución del conocimiento del uso de las plantas según características de los informantes, uso de las plantas por grupos campesinos en la franja tropical del Parque Nacional Natural Paramillo y utilidad del valor de uso en etnobotánica, estudio en el departamento de Putumayo, entre otros; por ello surge la necesidad de realizar más estudios en todo el territorio colombiano, ya que muchas de estas comunidades actualmente están siendo afectadas por actividades mineras, militares entre otras. Objetivo: Esta investigación fue realizada con el objetivo de rescatar el conocimiento etnobotánico. Metodología: Para la recopilación, clasificación y conservación de los saberes populares etnobotánicos, se emplearon herramientas como el modelo de ficha de plantas, entrevistas en las comunidades, grupos focales, fotografías, videos, audio y muestras taxonómicas. Resultados: se obtuvieron 44 muestras de plantas, con diferentes usos, con su respectivo nombre científico y su familia. El uso más común es el medicinal, la parte más utilizada es la hoja y la forma de preparación es decocción. Conclusión: En la actualidad, esta comunidad debe ser reasentada ya que recibe un impacto ambiental directo por el desprendimiento del polvillo de carbón y vibraciones por efecto de la voladura de las actividades mineras, afectando las fuentes hídricas superficiales, subterráneas y composición del suelo.

Palabras clave: Conocimiento Local, Etnobotánica, Grupos Focales, Plantas Medicinales, Saberes Populares, Vereda El Hatillo.

\begin{abstract}
Background: Ethnobotany studies the knowledge produced by the interaction between society and the use of plants that have been transmitted between generations and are still preserved in the different sectors of the rural community of the El Hatillo, Jurisdiction of La Loma de calentura, Town of El Paso, Cesar. Currently, very few ethnobotanical studies have been done in Colombia: ethnobotanical studies in the central Andes: Spread of knowledge about the use of plants by characteristics of the informants, use of plants
\end{abstract}


No. 2

by farmer groups in the tropical belt of Paramillo National Natural Park, and utility and value in use of ethnobotany, a study in the state of Putumayo, among others. Therefore, there is a need for further studies to be done throughout the Colombian territory, as many of these communities are currently being affected by mining and military activities among others. Objective: This research was conducted with the aim of rescuing the ethnobotanical knowledge. Methods: For collection, classification and preservation of popular ethnobotanical knowledge, tools like the plant spreadsheet model, community interviews, focus groups, pictures, videos, audio and taxonomic samples were used. Results: 44 samples of plants with different uses were obtained, each one with its own scientific name and family. The most common use was medicinal, the most used part is the leaf, and the way of preparation is cooking. Conclusion: Currently, this community must to be be relocated because it is affected by the direct environmental impact due to coal dust release as well as vibrations due to mining blast, which affects surface waters, groundwater and soil composition.

Keywords: Local Knowledge, Ethnobotany, Focus Groups, Medicinal Plants, Folk Knowledge, Community of El Hatillo.

\section{Resumo}

Antecedentes: A etnobotânica estuda simultaneamente as contribuições da botânica e da etnologia, evidenciando as interações entre as sociedades humanas e plantas como sistemas dinâmicos, assim como saberes transmitidos de uma geração a outra e que ainda se conservam nos diferentes setores da comunidade rural de "El Hatillo", Jurisdição "La Loma de Calentura", Município de "El Paso", Estado do Cesar. Na atualidade os estudos etnobotânicos realizados na Colômbia são muito escassos, alguns tais como: Estudos etnobotânicos nos Andes Centrais: Distribuição do conhecimento do uso das plantas segundo características dos informantes, uso das plantas por grupos camponeses na faixa tropical do "Parque Nacional Natural Paramillo" e utilidade do valor de uso em etnobotânica, estudo no Estado do Putumayo, entre outros. Portanto, surge a necessidade de realizar mais estudos em todo o território colombiano, já que muitas destas comunidades atualmente estão sendo afetadas por atividades mineiras, militares entre outras. Objetivo: Esta pesquisa foi realizada com o objetivo de resgatar o conhecimento etnobotânico. Metodologia: Para a recopilação, classificação e conservação dos saberes populares etnobotânicos, se empregaram ferramentas como o modelo de arquivo de plantas, entrevistas nas comunidades, grupos focais, fotografias, vídeos, áudio e amostras taxonômicas. Resultados: Obtiveram-se 44 amostras de plantas, com diferentes usos, com seu respectivo nome científico e sua família. O uso mais comum é o medicinal, a parte mais utilizada é a folha e a forma de preparação é de cocção. Conclusão: Na atualidade, esta comunidade deve ser realocada já que recebe um impacto ambiental direto pelo desprendimento da poeira de carvão e vibrações por efeito das explosões das atividades mineiras, afetando as fontes hídricas superficiais, subterrâneas e composição do solo.

Palavras-chave: Conhecimento local, Etnobotânica, Grupos focais, Plantas medicinais, Saberes populares, Comunidade "El Hatillo".

\section{Introducción}

La vereda El Hatillo, está ubicada en el extremo nororiental del departamento del Cesar, país Colombia; forma parte de la llanura del Caribe, con una población de 656 habitantes, de los cuales el $40 \%$ son nativos y el $60 \%$ corresponden a la zona rural. Debido a la actividad carbonífera en la zona, este asentamiento deber ser reubicado ya que es receptor de un impacto ambiental negativo directo, que genera la pérdida del conocimiento ancestral sobre las funciones socioculturales, socio-medicinales, etnoalimenticias y ambientales de las plantas que hacen parte de estos ecosistemas, en 
consecuencias a las alteraciones de los suelos y cambio en las actividades propias de esta comunidad Afrodescendiente. Por consiguiente, es necesario rescatar la memoria cultural etnobotánica que hasta el momento ha sido transferida de generación en generación y que tiende a su desaparición si no es recopilada y divulgada [1].

El impacto generado por las mineras se debe al desmonte y descapote que se realizó en la década de los 80 . Además las voladuras, transporte interno y la emisión de partículas de polvillo de carbón son transportadas por el viento sobre las viviendas, cultivos, calles, fuentes hídricas, alterando la composición y estado propio de esta zona. (Figuras 1 y 2).

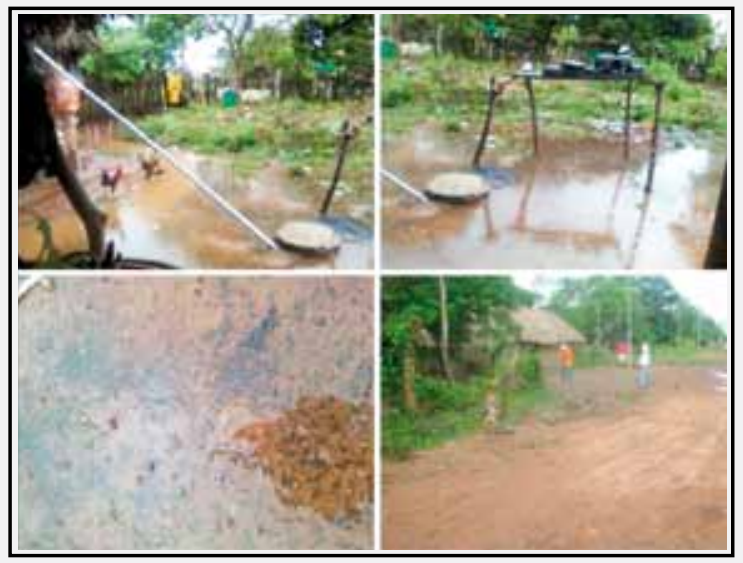

Figura 1. Condiciones físicas de la comunidad de El Hatillo. Fuente: Autores

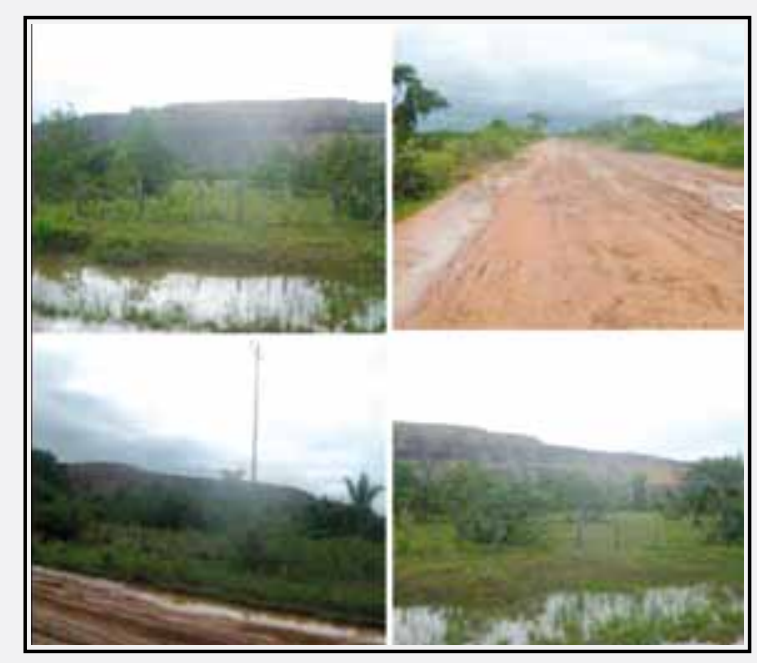

Figura 2. Visualización de los botaderos de la mina, desde la vereda El Hatillo.

Fuente: Autores
Cada día se presta más atención al estudio de las plantas medicinales de forma que la etnobotánica, la fitoterapia y la fitoquímica, tanto en la práctica de la medicina complementaria como en el ámbito académico [2]. El $80 \%$ de la población mundial, más de cuatro mil millones de personas, utiliza las plantas como principal remedio medicinal, según señala en [3]. Esta práctica está asociada al empirismo en muchos casos y faltan estudios químicos, clínicos y epidemiológicos que confirmen de forma fehaciente los efectos fisiológicos de las plantas y los principios activos responsables [3], [4], [5]. No hay que olvidar que el $25 \%$ de los fármacos existentes se obtienen de extractos vegetales o bien se han sintetizado a partir de sustancias halladas en la investigación fitoquímica. Las sustancias como la digitalina, la lidocaína, la pilocarpina o la dihidroergocristina son ejemplos de fármacos de uso común que pocas veces se asocian con su origen vegetal [2].

Hoy en día es, precisamente en los países del tercer mundo, donde la medicina tradicional sobrevive de una forma más auténtica y esto hace más fácil en dichos países identificar las plantas que necesitan ser científicamente evaluadas. Parece que la distancia entre la medicina tradicional y la ortodoxa empieza a acortarse y que ya no se considera la primera como un obstáculo del progreso científico. Un ejemplo claro es el estudio realizado en Camagüey, Cuba [2], del cual se obtuvo información etnobotánica sobre 111 especies vegetales pertenecientes a 96 géneros y 55 familias, con 173 indicaciones de uso medicinal para tratar afecciones respiratorias, digestivas, hepatobiliares y dermatológicas en siete comunidades. Estos datos empíricos obtenidos estimularon la validación farmacológica y toxicológica de algunas plantas [6]. Por otro lado, el Ministerio de Salud Pública de Cuba tiene un Programa de Investigaciones de Medicinas Tradicionales, aprobado en 1986, para el estudio de las plantas medicinales más utilizadas por la población y evaluar con métodos científicos actuales sus efectos farmacológicos y tóxicos. Ello ha
Julio - Diciembre 2015 ISSN 0122-820X E-ISSN 2422-5053 PP: $73-81$ 
No. 2

Julio - Diciembre 2015 ISSN 0122-820X E-ISSN 2422-5053 PP: $73-81$

76 permitido incorporar a la llamada medicina moderna los medios medicinales tradicionales con verdadera efectividad, ganando prestigio en la práctica médica actual [2].

La Organización Mundial de la Salud (OMS) estructuró en 1985 el Programa de Medicina tradicional Herbolaria, reconociendo la existencia de sustancias de origen vegetal que pueden considerarse fármacos importantes, útiles en más de 60 categorías terapéuticas y obtenidas principalmente de 91 especies.

La etnobotánica en Colombia es una ciencia relativamente nueva. Su desarrollo se inicia a partir de 1860 con la obra de "Botánica Indígena" de Florentino Vezga. A partir de entonces, se han llevado a cabo otras investigaciones principalmente con las comunidades indígenas del país con el fin de realizar inventarios de flora útil con predominio de plantas medicinales, alimenticias, rituales y para vivienda. Vale la pena destacar la obra "Flora Medicinal de Colombia" [6], [7], [8] de [9]. Posteriormente, se han realizado numerosas investigaciones en otros sectores de la población colombiana que incluyen comunidades campesinas, plazas de mercado de grandes ciudades, entre otros, con frecuencia culturales sobre el uso de los recursos vegetales [10].

\section{Materiales y métodos}

Área de estudio: está ubicada en el extremo nororiental de Colombia, en el departamento del Cesar, con una posición geográfica enmarcada entre las coordenadas $07^{\circ} 41^{\prime} 16^{\prime \prime} \mathrm{N}$ y $10^{\circ} 52^{\prime} 14^{\prime \prime} \mathrm{N}$ y $72^{\circ} 53$ '27'O y $74^{\circ} 08^{\prime} 28^{\prime \prime}$ O. Limita al norte con los departamentos de Magdalena y La Guajira, al este con el Norte de Santander y la República de Venezuela, al sur con el Norte de Santander y Santander, y al oeste con Bolívar y Magdalena (Figura 3). La vereda El Hatillo, jurisdicción de La Loma de Calenturas, Municipio de El Paso posee una población de 656 habitantes.

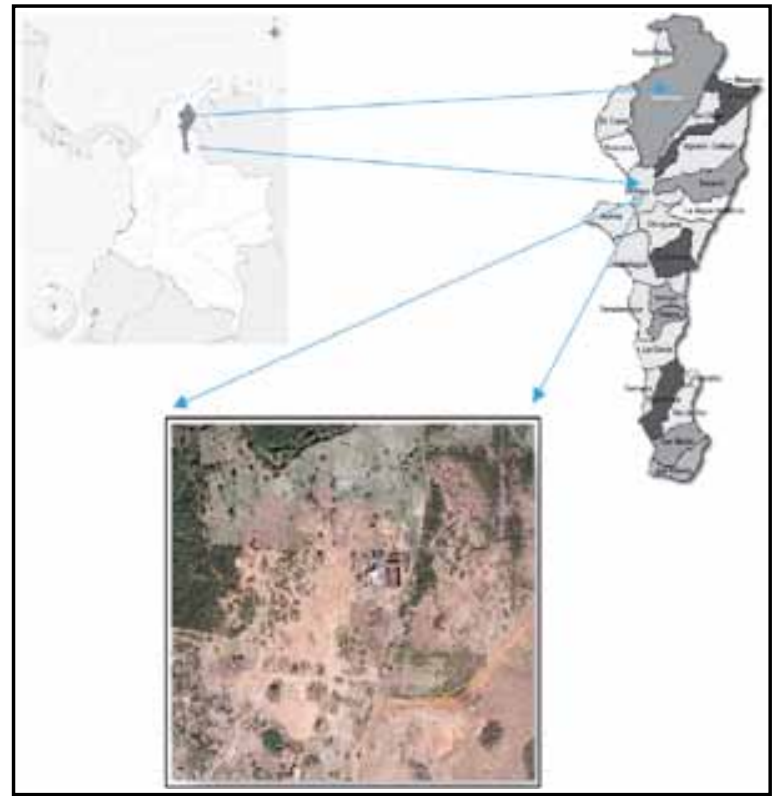

Figura 3. Localización del área de estudio. Fuente: Autores

La caracterización biofísica se realizó con base en los resultados obtenidos del trabajo de campo en la vereda El Hatillo y bibliografía específica [11].

Para la recopilación, clasificación $\mathrm{y}$ conservación de los saberes populares etnobotánicos, se emplearon herramientas como el modelo de ficha de plantas, entrevista en las comunidades [12], [13], grupos focales propuesta por [14], [15], fotografías, videos, audio y muestras taxonómicas. Se establecieron dos grupos focales (Figura 4), siguiendo la metodología propuesta por [13] y [14], que consiste en reunir a un grupo de personas de diferentes edades (adultos mayores, adultos, jóvenes y niños) para debatir y exponer acerca del desarrollo considerando el antes y el después de la llegada de la minería y el manejo etnobotánico con diferentes usos o fines, como medicinales, ornamentales $y$ alimenticios.

En el primer grupo focal se reunieron 27 personas de la comunidad, dentro de las cuales 16 corresponden a adultos mayores con un rango de edad de 60-90 años y 11 adultos con edades de 30-59 años. Para el segundo grupo focal se reunieron 43 personas; estaba integrado por 24 jóvenes de edad entre 1629 años y 19 niños con edad entre 10-15 
años. Para la recolección de información se emplearon entrevistas semiestructuradas realizadas a adultos mayores, adultos, jóvenes e informantes claves y talleres didácticos aplicados a niños (Figura 4).

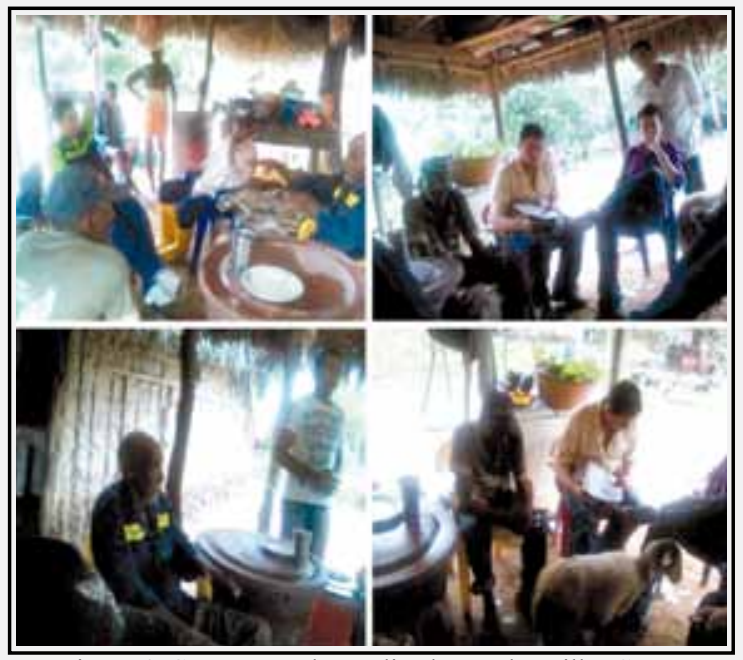

Figura 4. Grupos Focales realizado en E1 Hatillo, Cesar, Noviembre 6 de 2010.

Fuente: Autores

Como técnica de registro de información los registros de este estudio quedaron depositadas en el herbario del semillero de investigación de Ecología Humana (SIECH) de la Fundación del Área Andina sede Valledupar. [16]. Las plantas recolectadas en la comunidad de El Hatillo fueron secadas, prensadas y organizadas de acuerdo al nombre común y uso dado por la comunidad Hatillera, se realizó un estudio bibliográfico propuesto por [17] y [18]. Por último, se utilizaron claves taxonómicas [19], para determinar el nombre científico de las plantas recolectadas.

\section{Resultados y análisis}

Como resultado del trabajo de campo realizado en la Vereda de El Hatillo se logró conocer la importancia de las relaciones existentes entre el campesino y su entorno, específicamente con la flora, a través de la cual se obtienen ingresos económicos, alimentos, productos medicinales entre otros beneficios.

El conocimiento de las plantas en la comunidad de la Vereda El Hatillo es bastante amplio, desde niños con edades de 9 años hasta adultos mayores con edades de hasta 86 años conocen e interpretan los saberes etnobotánicos, transmitidos de generación en generación, principalmente usados por aquellas personas que sobrepasan los 60 años de edad. Éstas personas fueron consideradas informantes claves, a pesar de que fue un grupo pequeño con sólo 16 personas, sus aportes fueron significativos; el grupo estuvo conformado por nativos que han tenido siempre una relación directa y continua con las plantas manifestado su interés en la salud de la comunidad.

En resumen, la información recopilada indica que son 44 especies vegetales las que la población emplea de modo diverso (integran 28 familias botánicas): Se señalaron cuatro usos: medicinal, maderable, para alimentación y para aseo personal. El uso más común fue medicinal con 39 especies; la parte más utilizada de la planta es la hoja y ello fue indicado para 32 especies; la forma de preparación más frecuente es la cocción, empleada en el uso de 34 especies (Tabla I). La comunidad de El Hatillo las ha utilizado por aproximadamente 160 años, cuentan con un amplio estudio sobre su efecto medicinal. Una de las plantas más utilizadas por la comunidad es la "malva" ya que la utilizaban para varios fines. Ellos comentaron "Nosotro', en aquella época, coijiamo una matica que nos ayudaba a jalá el pescao', eso pa' l barbul, era efe' tivo, esa matica por acá se le llama Malva".

Otras de las especies más utilizadas son el toronjil, el guarumo y el totumo, ellos contaron que "vea u'te' coge el toronjil, el guarumo, el totumo, to'as estas matas son para cura gripa, o cuando uno está cojeó de los bronquios".

En la Tabla I, se muestra el listado de las plantas utilizadas por la comunidad de El Haltillo; se detalla para cada una el nombre común, el nombre científico, la familia a la que pertenece, los usos, la parte utilizada y la forma de preparación como ilustración de la forma en que se organizó la información proveniente de cada uno de los subgrupos con que se realizó el trabajo dentro de la vereda [20], [21], [22], [23], [24], [25], [26], [27].
Julio - Diciembre 2015 ISSN 0122-820X E-ISSN 2422-5053 PP: $73-81$ 
Respuestas

Cúcuta-Colombia

Vol. 20

No. 2

Julio - Diciembre 2015

ISSN 0122-820X

E-ISSN 2422-5053

PP: 73-81
En el gráfico de las tablas número 5, 6 y 7 se puede evidenciar que el uso más común es el medicinal, la parte más utilizada de la planta es la hoja, y la forma de preparación de mayor frecuencia es de cocción y cruda.

Según relata el señor Rumaldo Parra, el último de los hermanos Parra que queda vivo, el Hatillo... "era toda montaña, pura montaña inmensa. En tiempo de invierno, era tire pa allá, tire pa acá..."

En ese entonces era cazadores. El señor Rumaldo Parra dice "Uff, como no. Casábamos venados, eso se acabó; y armadillos...”. También eran pescadores antes y como el mismo cuenta "Uff, cuando habian pescados...."

Tabla I. Plantas de uso común en la comunidad de El Hatillo.

\begin{tabular}{|c|c|c|c|c|c|}
\hline NOMBRE COMÚN & NOMBRE CIENTÍFICO & FAMILIA & USO & PARTE UTILIZADA & $\begin{array}{c}\text { FORMA DE } \\
\text { PREPARACIÓN }\end{array}$ \\
\hline Paico & Chenopodiun ambrosioides & Amaranthaceae & Medicinal & Hoja & Decocción \\
\hline Mango & Mangifera indica & Anacardiaceae & Medicinal & Fruto & Decocción y cruda \\
\hline Marañón & Anacardium occidental & Anacardiaceae & Medicinal & Fruto & Decocción \\
\hline Guanábana & Annona muricata & Annonaceae & Medicinal & Fruto y la hoja & Decocción y crudo \\
\hline Lengua de suegra & Sanseviera trifasciata & Asparagaceae & Medicinal & Hoja & Decocción \\
\hline Caléndula & Calendula officinalis & Asteraceae & Medicinal & Hoja & Decocción \\
\hline Stevia & Stevia rebaudiana Bertoni & Asteraceae & Medicinal & Hoja & Decocción y crudo seco \\
\hline Manzanilla & Matricaria chamomilla. & Asteraceae & Medicinal & Hoja & Decocción \\
\hline Totumo & Crescentia cujete & Bignoniaceae & Medicinal & Fruto & Decocción \\
\hline Achiote & Bixa Orellana. & Bixaceae & Medicinal & Hoja & Crudo \\
\hline Ceiba roja & Ceiba pentandra (L.) Gaertn & Bombacaceae & Madera & Tronco y ramas & Ebanistería \\
\hline Esponjilla & Lagenaria vulgaris & Cucurbitaceae & Aseo personal & Fruto & Crudo \\
\hline Balsamina & Momordica charantia & Cucurbitaceae & Medicinal & Toda la planta & Decocción \\
\hline Cola de caballo & Equisetum arvense & Equisetaceae & Medicinal & Hoja & Decocción \\
\hline Bálsamo & Saxifraga geranoides & Fabaceae & Medicinal & Hoja & Decocción \\
\hline Tolu & Myroxylon toluiferum & Fabaceae & Madera & Tronco y ramas & Ebanistería \\
\hline Sen & Cassia angustifolia & Fabaceae & Medicinal & Hoja & Decocción \\
\hline Matarraton & Bocconia frutescens & Fabaceae & Medicinal & Hoja & Decocción y crudo seco \\
\hline Roble & Quercus robur & Fagaceae & Madera & Tronco y ramas & Ebanistería \\
\hline Lirio & Iris germánica & Iridaceae & Medicinal & Raíz & Decocción \\
\hline Toronjil & Melissa officinalis & Lamiaceae & Medicinal & Hoja & Decocción \\
\hline Orégano & Origanun vulgare & Lamiaceae & Medicinal & Hoja & Decocción \\
\hline Albahaca & Ocimun basilicum & Lamiaceae & Medicinal & Hoja & Decocción \\
\hline Hierba buena & Mentha sativa & Lamiaceae & Medicinal & Hoja & Decocción \\
\hline Salvia & Salvia officinalis & Lamiaceae & Medicinal & Hoja & Decocción \\
\hline Escobilla & Sida cordifolia & Malvaceae & Medicinal & Hoja & Decocción y crudo seco \\
\hline Malva & Malva sylvestris & Malvaceae & Medicinal & Hoja & Decocción y Crudo \\
\hline Guasimo & Guazuma ulmifolia & Malvaceae & Medicinal & Hoja & Decocción y crudo seco \\
\hline Cedro & Cedrela odorata & Meliaceae & Madera & Tronco y ramas & Ebanistería \\
\hline Guáimaro & Brosimun alicastrum & Moraceae & Medicinal & Fruto & Decocción \\
\hline Guineo & Musa acuminata & Musaceae & Medicinal & Fruto & Crudo \\
\hline Guayabita sabanera & Psidium guajava & Myrtaceae & Fruto y medicinal & Toda la planta & Decocción y cruda \\
\hline Eucalipto & Eucalyptus globosus & Myrtaceae & Medicinal & Hoja & Decocción \\
\hline Flor escondida & Phyllanthus niruri & Phyllantaceae & Medicinal & Toda la planta & Decocción \\
\hline Llantén & Plantag omajor & Plantaginaceae & Medicinal & Hoja & Decocción \\
\hline Cadillo & Acaena splendens & Rosaceae & Medicinal & Hoja & Decocción y crudo seco \\
\hline Naranja agria & Citrus aurantium var. Amara & Rutaceae & Medicinal & Hoja & Decocción y crudo seco \\
\hline Mamón & Melicoccus bijugatus & Sapindaceae & Medicinal & Fruto & Decocción y cruda \\
\hline Zarzaparrilla & Smilax aspera & Smilariaceae & Medicinal & Toda la planta & Decocción \\
\hline Zarza hueca & Byttneria aculeata (Jacq.) Jacq. & Sterculiaceae & Medicinal & Hoja & Decocción y crudo seco \\
\hline Guarumo & Cecropia peltata & Urticaceae & Medicinal & Hoja & Decocción \\
\hline Cedrón & Lippia citriodora & Verbenaceae & Medicinal & Toda la planta & Decocción \\
\hline Venturosa & Lantana cámara & Verbenaceae & Medicinal & Hoja & Decocción y crudo seco \\
\hline Sábila & Aloe vera & Xanthorrhoeaceae & Medicinal & Toda la planta & Crudo y decocción \\
\hline
\end{tabular}




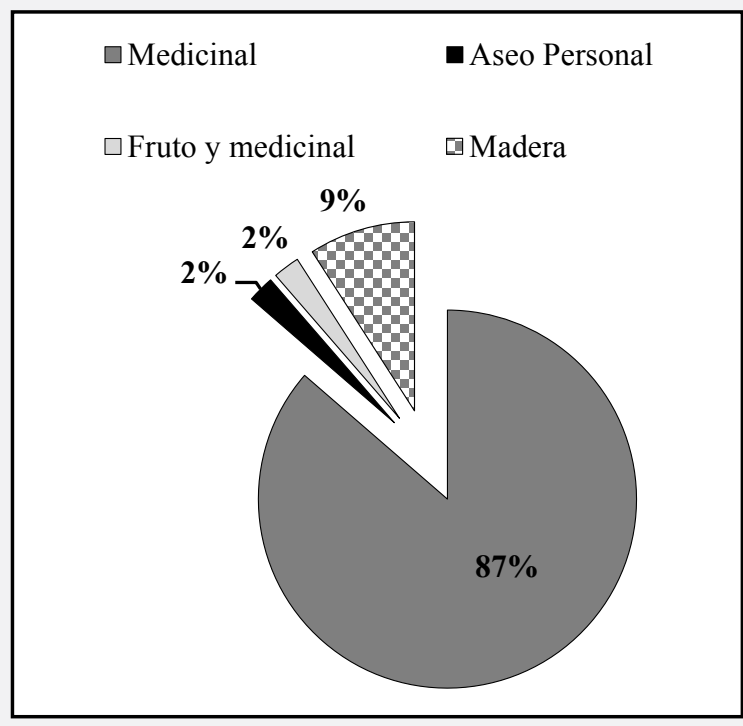

Figura 5. Porcentajes de los usos de las plantas medicinales. Fuente: Autores

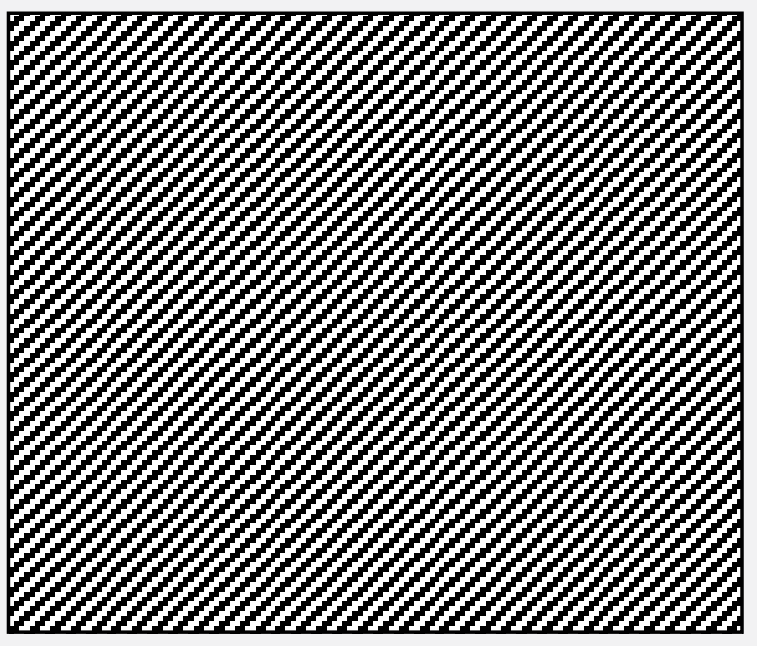

Figura 6. Porcentajes de las partes más utilizadas de las plantas medicinales.

Fuente: Autores

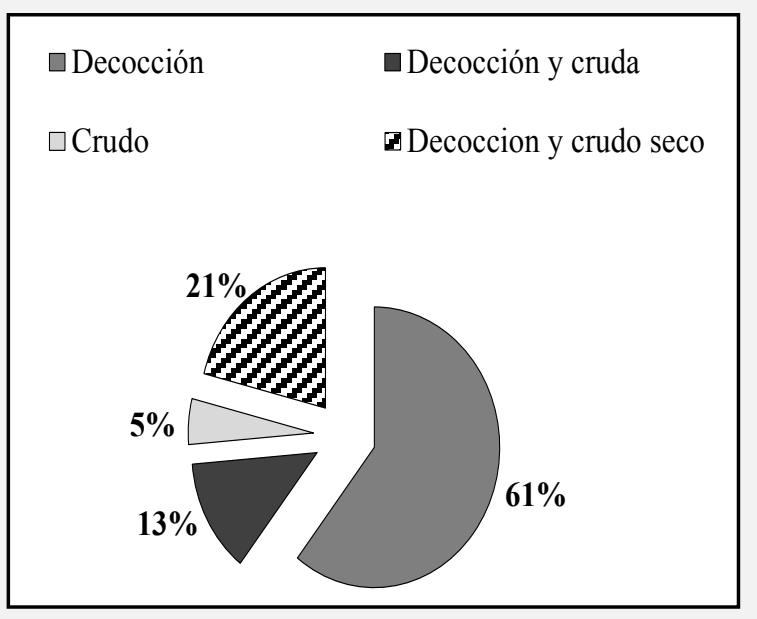

Figura 7. Forma de preparación de las plantas medicinales. Fuente: Autores
En la clasificación dada frente a la pesca el señor Candelario nos dice: "Uno llegaba allá a la orilla y tiraba un mantazo en la orilla del rio y tenía que llamar dos o tres pa poder jalar la atarralla acá a la playa, eran 200 o 300 pescados de así de este grande", señalando con su mano hasta más arriba del codo.0La señora Ana cuenta "Yo taba contando que nosotros tamos pasando cosas pero antes; no, nosotros nos criamos pisando la comida. Se veía el galápago, las tortugas, aquí había comida de todo, en cambio ahora ya no ya todo eso se acabó. Había más animales porque había masa monte, si tiene que ser. La carne no hacía falta e la casa, pa' tener la liga".

El señor Candelario relata cómo se quemaban los montes: "Si, acabaron con el monte. Habia micos...".Su esposa cuenta que aqui habia hasta tigre." También tenían hasta cabuya, plátano y todo lo de comida....."

El señor Rumaldo cuenta que "las gallinas y los marranos nunca faltaban".

\section{Conclusiones}

Los estudios etnobotánicos son una herramienta valiosa para acercar más profundamente a las ciencias biológicas desde la perspectiva de quienes con viven con la naturaleza ya que tienen mucho que enseñar y aportar al campo investigativo sobre el uso y empleo de las plantas. A través de este trabajo se puede ver la inmensa riqueza de los saberes populares que han pasado de generación en generación y permanecen aún en nuestros pueblos, que merecen una pronta atención frente a la pérdida acelerada de estos conocimientos ancestrales sobre las funciones socioculturales, socio-medicinales, etnoalimenticias y ambientales de las plantas que hacen parte de estos ecosistemas entre otras, generados por los desplazamientos, reasentamientos humanos y destrucción de la flora producto de la actividad minera. 
No. 2

Julio - Diciembre 2015 ISSN 0122-820X E-ISSN 2422-5053 PP: 73-81
En la Vereda EL Hatillo jurisdicción La Loma, cabecera municipal El Paso, se estudiaron 44 especies vegetales correspondientes a 28 familias, los usos fueron 4: el medicinal, el maderable, para alimentación y para aseo personal, el uso más común fue el medicinal con 39 especies, la parte más utilizada de la planta es la hoja con 32 especies, la forma de preparación más frecuente es la cocción, con 34 especies.

Los impactos causados por las mineras han generado un deterioro socio-ambiental irreversible en la vereda El Hatillo, desde el desmonte y descapote que dio inicio hacia la década de los ochenta hasta nuestros días, disminuyendo la diversidad de plantas en la zona y como consecuencia el desarraigo de saberes etnobotánicos que han trascendido de generación en generación. Actualmente una de las preocupaciones que se vive en esta comunidad étnica afrocolombianos, es el deterioro de estos saberes, por lo que debe ser reasentada ya que es receptor de un impacto ambiental negativo directo.

\section{Agradecimientos}

Los autores desean expresar sus agradecimientos a la Fundación Universitaria del área Andina por su apoyo financiero y logístico; a Jerson Díaz Rivero, Abel Carreño, Luis Galván, Sergio Maestre y Nicolás Daza, integrantes del semillero de investigación de Ecología Humana-SIECH; a todas las personas entrevistadas, las cuales tuvieron la bondad de brindarnos su tiempo y aportarnos sus valiosos conocimientos y a quienes con su entusiasmo y avidez investigativa hicieron posible este estudio.

\section{Referencias}

[1] J. Hurrell, "Las posibilidades de la etnobotánica y un nuevo enfoque a partir de la ecología y su propuesta cibernética", Revista Española de Antropología Americana, vol. 17, pp. 235-257, 1987.
[2] A. Beyra, M. León, E. Iglesia, et. al, "Estudios etnobotánicos sobre plantas medicinales en la provincia de Camagüey (Cuba)", Anales del Jardín Botánico de Madrid, vol. 61, no. 2, pp.185-203, 2004. [Online]. Disponible en: http://www.redalyc.org/ pdf/556/55661207.pdf.

[3] J. Pauro, F. Gonzalez, B. Gamarra, et. al, "Plantas alimenticias, medicinales y biocidas de las comunidades de Muñani y Suatia, provincia de Lampa (Puno Perú)", Ecología Aplicada, vol. 10, no. 1, pp. 41-49, 2011.

[4] P. Zela, "Plantas medicinales empleadas para el tratamiento de componentes sindrómicos de infecciones respiratorias", Tesis para optar el título profesional de Enfermera, Universidad Nacional del Altiplano, Puno, Perú, 2001.

[5] C. Chifa. Plantas usadas en medicina tradicional, aspectos fitoquímicos y farmacológicos. Segundo Curso Internacional de Plantas Medicinales. Publicación del Instituto de Fitoterapia Americano. 2001.

[6] H. García. Flora medicinal de Colombia. Botánica médica. Segunda edición. Tomo I. Santafé de Bogotá: Tercer mundo editores. 1974.

[7] H. García. Flora medicinal de Colombia. Botánica médica. Segunda edición. Tomo II. Santafé de Bogotá: Tercer mundo editores. 1974.

[8] H. K. Sharma, L. Chhangte y K. Dolui, "Traditional medicinal plants in Mizoran, India", Fitoterapia, vol. 72, no. 2, pp. 146-161.

[9] H. García. Flora medicinal de Colombia. Botánica médica. Segunda edición. Tomo III. Santafé de Bogotá: Tercer mundo editores. 1974. 
[10] G. Zuluaga. El aprendizaje de las plantas en la senda de un camino olvidado. Santafé de Bogotá: Seguros Bolívar, 1994.

[11] B. Gonzáles, M. Mora, y M. Clavijo, "Estudios etnobotánicos de las plantas medicinales empleadas por la comunidad de Zaque-municipio de Gacheta Cundinamarca", Revista Tecne, Episteme y Didaxis, no. 9, pp. 35-43, 2001.

[12] Carvajal. Historia del Hatillo. Colombia, Pp.:73, 2004.

[13] L. Mulet, "Estudio etnobotánico en la provincia de Castellon", Natura Medicatrix, no. 37-38, pp. 22-29, 1995.

[14] Martin, G.J., Hoare, A.L. \& Posey, D.A. 1996. The discussion fo-cuses on how to recognize the intellectual contribution of lo-cal people. People and Plant Working Paper 2, UNESCO,París.

[15] R. Sampieri, L. Fernández \& L. Batista. Libro metodología de la investigación. Colombia: McGraw-Hill, 2004.

[16] J. Cáceres, A. Wesley y A. Longman. Técnicas de Investigación en Sociedad, Cultura y Comunicación. Primera Edición. México: Editorial Addison Wesley Longman, 1998.

[17] R. Fonnegra y S. L. Jiménez Plantas medicinales aprobadas en Colombia. Colombia: Editorial Universidad de Antioquia, 2007.

[18] Fundación Chemonisc Colombia. Manual de fitoprotección y análisis de plaguicidas. 2003, pp. 4-12.

[19] A. Marzocca, Nociones básicas de taxonomía vegetal, $1^{\mathrm{a}}$ edición. Costa Rica: IICA, 1985.

[20] A.Fernandes, "Farmacognosia". Volume
III. En Farmacognosia Experimental. Lisboa: Fundaçao Calouste Gulbenkian, 1982.

[21] S. Altschul. La investigación del herbario. México, pp. 70-78, 1977.

[22] J. Carmona, R. Gil y M. Rodríguez, "Descripción taxonómica, morfológica y etnobotánica de 26 hierbas comunes que Crecen en la ciudad de MéridaVenezuela", Boletín Antropológico, vol. 26, no. 73, 2008.

[23] J. Fernández, \& R. Yepes. Curso de herbologia y Alelopatía 305698. Universidad Nacional Abierta y a Distancia.. 2013. pp. 1-227. [Online]. Disponible en: http://datateca. unad.edu.co/contenidos/305698/ M O D U L O _ H E R B O L O G I A ALEOPATIA_305698_2013-ver2.pdf.

[24] W. N. Richardson, y T. Stubbs. Plants, Agriculture \& Human Society. W.A. Benjamin. Menlo Park, California: University of California, pp 353, 1978.

[25] F. J. Lipp, "Methods for Ethnopharmacological field work", Journal of Ethnopharmacology, vol. 25, no. 2, pp. 139-150, 1989.

[26] M. Balick, y P. Cox. Plants, People and Culture: The Science of Ethnobotany. Nueva York: Scientific American, 1996. ISSN 0122-820X ISSN 2422-5053 73-81 